\title{
INCOMPLETE INTERVAL FUZZY PREFERENCE RELATIONS FOR SUPPLIER SELECTION IN SUPPLY CHAIN MANAGEMENT
}

\author{
Yejun XU', Ravi PATNAYAKUNI', Feifei TAO ${ }^{c}$, Huimin WANG ${ }^{\mathrm{d}}$ \\ ${ }^{\mathrm{a}, \mathrm{d}}$ State Key Laboratory of Hydrology-Water Resources and Hydraulic Engineering, Hohai University, \\ Nanjing, 210098 Jiangsu, P.R. China \\ ${ }^{a}, \mathrm{c}, \mathrm{d}$ Business School, HoHai University, Nanjing, 211100 Jiangsu, P.R. China \\ ${ }^{\mathrm{b}}$ Department of Economics and IS, University of Alabama in Huntsville, 35763 Huntsville, USA
}

Received 28 July 2012; accepted 20 January 2013

\begin{abstract}
In the analytical hierarchy process (AHP), it needs the decision maker to establish a pairwise comparison matrix requires $n(n-1) / 2$ judgments for a level with $n$ criteria (or alternatives). In some instances, the decision maker may have to deal with the problems in which only partial information and uncertain preference relation is available. Consequently, the decision maker may provide interval fuzzy preference relation with incomplete information. In this paper, we focus our attention on the investigation of incomplete interval fuzzy preference relation. We first extend a characterization to the interval fuzzy preference relation which is based on the additive transitivity property. Using the characterization, we propose a method to construct interval additive consistent fuzzy preference relations from a set of $n-1$ preference data. The study reveals that the proposed method can not only alleviate the comparisons, but also ensure interval preference relations with the additive consistent property. We also develop a novel procedure to deal with the analytic hierarchy problem for group decision making with incomplete interval fuzzy preference relations. Finally, a numerical example is illustrated and a supplier selection case in supply chain management is investigated using the proposed method.
\end{abstract}

Keywords: incomplete interval fuzzy preference relation, additive consistent, group decision making, supplier selection.

JEL Classification: A12, C44, C80, D81.

Corresponding author Yejun Xu

E-mail:xuyejohn@163.com 


\section{Introduction}

Analytic hierarchy process (AHP), as a multiple criteria decision making (MADM) tool and a weight estimation technique, has been extensively applied in many areas such as selection, evaluation, planning and development, decision making, forecasting, and so on (Vaidya, Kumar 2006). The conventional AHP requires exact judgments. However, the decision maker may not estimate his/her preference with exact numerical values, but with interval number due to the increasing complexity and uncertainty of real-life decision making problem, and the decision maker's limited attention and information processing capabilities. In such situations, an interval preference relation is very suitable for expressing the decision maker's uncertain preference information. Since the preference information is usually expressed in two formats: multiplicative and fuzzy preference relations. The interval preference relations also have the two forms. Saaty and Vargas (1987) presented interval judgments as a way to model subjective uncertainty. Arbel (1989) viewed interval judgments as linear constraints on weights and formulated the weight estimation problem as a linear programming (LP) model, which was found by Kress (1991) infeasible for inconsistent interval comparison matrices. Islam et al. (1997) used Lexicographic Goal Programming (LGP) to find out weights from pairwise inconsistent interval judgment matrices, and explored a number of properties and advantages of LGP as a weight estimation technique. The LGP method was recently found flawed by Wang (2006). Mikhailov (2002) proposed a fuzzy preference programming (FPP) method and its variant, to derive crisp priorities from interval comparison matrices. Wang et al. (2005) proposed a two-stage logarithmic goal programming (TLGP) method to generate weights from interval comparison matrices, which can be either consistent or inconsistent. Wang and Chin (2006) also proposed an eigenvector method (EM) to generate interval or fuzzy weight estimate from an interval or fuzzy comparison matrix which differs from Csutora and Buckley's (2001) Lambda-Max method in several aspects. Wang and Elhag (2007) very recently suggested a goal programming method (GPM) for obtaining normalized interval weights from an interval comparison. The GPM turns out to be also applicable to crisp comparison matrices.

About the interval fuzzy preference relation, Xu (2004a) defined the concept of compatibility degree of two interval fuzzy preference relations, and showed the compatibility relationship among individual interval fuzzy preference relations and collective interval fuzzy preference relation. Herrera et al. (2005) developed an aggregation process for combining interval fuzzy preference relations with other types of information such as numerical preference relation and linguistic preference relation. Jiang (2007) gave an index to measure the similarity degree of two interval fuzzy preference relations, and used the error-propagation principle to determine the priority vector of the aggregated interval fuzzy preference relations. Recently, $\mathrm{Xu}$ and Chen (2008) established some linear programming models for deriving the priority weights from various interval fuzzy preference relations.

All the above researches focused on the studies of the preference relations with complete information. A complete preference of order $n$ necessitates the completion of all $n(n-1) / 2$ judgments in its entire top triangular portion. Sometime, however, a decision maker (DM) may develop a preference relation with incomplete information because of 
(1) time pressure, lack of knowledge, and the DM's limited expertise related with problem domain (Chiclana et al. 2008; Liu et al. 2012; Xu et al. 2009, 2010, 2013; Xu 2004b, 2005; $\mathrm{Xu}$, Chen 2008); (2) when the number of the alternatives, $n$, is large. In such cases it may be practically impossible, or at least unacceptable from the point of view of the decision maker, to perform all the $n(n-1) / 2$ required comparisons to complete the pairwise comparison matrices (Fedrizzi, Silvio 2007); (3) it can be convenient/necessary to skip some direct critical comparison between alternatives, even if the total number of alternatives is small (Fedrizzi, Silvio 2007); and (4) an expert would not be able to efficiently express any kind of preference degree between two or more of the available options. This may be due to an expert not possessing a precise or sufficient level of knowledge of part of the problem, or because that expert is unable to discriminate the degree to which some options are better than others (Alonso et al. 2008; Herrera-Viedma et al. 2007a, b). Up to now, some related theory studies with incomplete preference relations have been given. Nevertheless, there are only few researches which concern the incomplete interval fuzzy preference relation. Therefore, it is necessary to pay attention to this issue. In order to do this, this rest of the paper is structured in the following way. In Section 2, we first review the traditional multiplicative preference relation and fuzzy preference and their transformation function. Then, we extend the proposition which was introduced by Herrera-Viedma et al. (2004). In Section 3, we introduced the concept of interval multiplicative consistent preference relation, interval additive consistent preference relation, and their transformation function. We also present some useful results which are based on the additive consistent. In Section 4, we present the incomplete interval additive preference relation. We develop a simple and practical method for constructing a complete additive consistent interval preference relation. We also develop a novel procedure to deal with the analytic hierarchy problem for group decision making with incomplete interval fuzzy preference relations. In Section 5, a numerical example is illustrated and a supplier selection case in supply chain management is investigated using the proposed method. This paper is concluded in the final section.

\section{Preliminaries}

Let $X=\left\{x_{1}, x_{2}, \ldots, x_{n}\right\} \quad(n \geq 2)$ be a finite set of alternatives, where $x_{i}$ denotes the $i$ th alternative. In the multiple attribute decision making problems, the decision maker needs to rank the alternatives $x_{1}, x_{2}, \ldots, x_{n}$ from the best to the worst according to the preference information. A brief description of the multiplicative preference relation and fuzzy preference relation is given below.

The multiplicative preference relation is a positive preference relation $A \subset X \times X$, $A=\left(a_{i j}\right)_{n \times n}$, where $a_{i j}$ denotes the relative weight of alternative $x_{i}$ with respect to $x_{j}$. The measurement of $a_{i j}$ is described using a ratio scale and in particular, as shown by Saaty (1980), $a_{i j} \in\{1 / 9,1 / 8, \ldots, 1,2, \ldots, 9\}: a_{i j}=1$ denotes the indifference between $x_{i}$ and $x_{j}, a_{i j}=9$ (or $a_{j i}=1 / 9$ ) denotes that $x_{i}$ is unanimously preferred to $x_{j}$, and $a_{i j} \in\{2,3, \ldots, 8\}$ denotes the intermediate evaluations. It is multiplicative reciprocal, i.e. $a_{i j} a_{j i}=1, \forall i, j \in\{1,2, \ldots, n\}$ and in particular, $a_{i i}=1, \forall i \in\{1,2, \ldots, n\}$. Thus we have the following definition. 
Definition 1. Let $A=\left[a_{i j}\right]_{n \times n}$ be a multiplicative preference relation, then $A$ is called a consistent multiplicative preference relation (or called consistent reciprocal judgment matrix (Saaty 1980)), if $a_{i j}=a_{i k} a_{k j}$, for all $i, j, k$.

The fuzzy preference relation $R$ is described as follows: $R \subset X \times X, R=\left[r_{i j}\right]_{n \times n}$, with membership function $u_{R}: X \times X \rightarrow[0,1]$, where $u_{R}\left(x_{i}, x_{j}\right)=r_{i j}$ denotes the preference degree of the alternative $x_{i}$ over $x_{j}$ (Kacprzyk 1986; Tanino 1984): $r_{i j}=0.5$ denotes indifference between $x_{i}$ and $x_{j}, r_{i j}=1$, denotes that $x_{i}$ is unanimously preferred to $x_{j}$, and $0.5<r_{i j}<1$ (or $0<r_{j i}<0.5$ ) denotes that $x_{i}$ is preferred to $x_{j}$.

Definition 2. Let $R=\left[r_{i j}\right]_{n \times n}$ be a preference relation, then $R$ is called a fuzzy preference relation, if:

$$
r_{i j} \in[0,1], r_{i j}+r_{j i}=1, r_{i i}=0.5 \text {, for all } i, j=1,2, \ldots, n .
$$

Herrera-Viedma et al. (2004) studied the transformation function between reciprocal multiplicative preference relations with values in the interval scale $[1 / 9,9]$ and reciprocal fuzzy preference relations with values in $[0,1]$. They developed the propositions given below.

Proposition 1. Consider a set of alternatives $X=\left\{x_{1}, x_{2}, \ldots, x_{n}\right\}$, associated with a reciprocal multiplicative preference relation $A=\left[a_{i j}\right]_{n \times n}$ with $a_{i j} \in[1 / 9,9]$. Then, the corresponding reciprocal fuzzy preference relation, $R=\left[r_{i j}\right]_{n \times n}$, with $r_{i j} \in[0,1]$, associated with $A$ is given as follows:

$$
r_{i j}=g\left(a_{i j}\right)=\frac{1}{2}\left(1+\log _{9} a_{i j}\right) .
$$

Proposition 2. For a reciprocal fuzzy preference relation $R=\left[r_{i j}\right]_{n \times n}$, the following statements are equivalent:
(a) $r_{i j}+r_{j k}+r_{k i}=\frac{3}{2}, \forall i, j, k$;
(b) $r_{i j}+r_{j k}+r_{k i}=\frac{3}{2}, i<j<k$.

Proposition 3. For a reciprocal fuzzy preference relation $R=\left[r_{i j}\right]_{n \times n}$, the following statements are equivalent:
(a) $r_{i j}+r_{j k}+r_{k i}=\frac{3}{2}, i<j<k$;
(b) $r_{i(i+1)}+r_{(i+1)(i+2)}+\ldots+r_{(j-1) j}+r_{j i}=\frac{j-i+1}{2}, i<j$.

Herrera-Viedma et al. (2004) showed that Proposition 3 can be used to construct a consistent fuzzy preference relation from the set of $n-1$ values $\left\{r_{12}, r_{23}, \ldots, r_{n-1 n}\right\}$. The above propositions were also used by Wang and Chen (2007). In the following, we give a more general result.

Proposition 4. For a reciprocal fuzzy preference relation $R=\left[r_{i j}\right]_{n \times n}$, the following statements are equivalent:
(a) $r_{i j}+r_{j k}+r_{k i}=\frac{3}{2}, i<j<k$;
(b) $r_{i j_{1}}+r_{j_{1} j_{2}}+\ldots+r_{j_{t-1} j_{t}}+r_{j_{t}}=\frac{t+1}{2}$. 
Proof. $(a) \Rightarrow(b)$ we will use mathematical induction to prove this part of the proposition. The base clause it is obviously true for $k=1$, because it is reduced to the reciprocity property, which we are assuming (Definition 2). The recursion clause requires us to that if the hypothesis is true for $t=n$ :

$$
r_{i j_{1}}+r_{j_{1} j_{2}}+\ldots+r_{j_{n-2} j_{n-1}}+r_{j_{n-1} j_{n}}+r_{j_{n} i}=\frac{n+1}{2},
$$

then it is true for $t=n+1$ :

$$
\begin{gathered}
r_{i j_{1}}+r_{j_{1} j_{2}}+\ldots+r_{j_{n-1} j_{n}}+r_{j_{n} j}+r_{j i}=\left(r_{i j_{1}}+r_{j_{1} j_{2}}+\ldots+r_{j_{n-2} j_{n-1}}+r_{j_{n-1} j_{n}}\right)+r_{j_{n} j_{n+1}}+r_{j_{n+1} i}= \\
\quad \frac{n+1}{2}-r_{j_{n} i}+r_{j_{n} j_{n+1}}+r_{j_{n+1} i}=\frac{n-1}{2}+r_{i j_{n}}+r_{j_{n} j_{n+1}}+r_{j_{n+1} i}=\frac{n-1}{2}+\frac{3}{2}=\frac{n+2}{2} .
\end{gathered}
$$

So the result is established.

$(b) \Rightarrow(a)$.

$$
\begin{gathered}
r_{i j}+r_{j k}+r_{k i}=1-r_{j i}+1-r_{k j}+r_{k i}=2+\left(r_{i j_{1}}+\ldots+r_{j_{t-1} j}-\frac{t+1}{2}\right)+\left(r_{j j_{1}}+\ldots+r_{j_{t-1} k}-\frac{t+1}{2}\right)+r_{k i}= \\
1-t+\left(r_{i j_{1}}+\ldots+r_{j_{t-1} j}+r_{j j_{1}}+\ldots+r_{j_{t-1} k}+r_{k i}\right)=1-t+\frac{2 t+1}{2}=\frac{3}{2},
\end{gathered}
$$

which completes the proof.

From above, we know that the difference between Proposition 3 and Proposition 4 is that we only need know the values $r_{i j_{1}}, r_{j_{1} j_{2}}, \ldots, r_{j_{t-1} j_{t}}, r_{j_{t} i}$ in the Proposition 4 , and the differences of $j_{2}-j_{1}=j_{3}-j_{2}=\ldots=j_{t}-j_{t-1}$ do not need to equal to 1 , even they do not be equivalent, we only need the sequential values of $r_{i j_{1}}, r_{j_{1} j_{2}}, \ldots, r_{j_{t-1} j_{t}}, r_{j_{t}}$ (for example $r_{13}, r_{35}, r_{51}$ ). And also in the real situation, we do not just know the values $r_{12}, r_{23}, \ldots, r_{n-1 n}$. Proposition 3 is only a special case of Proposition 4.

We make note that, if the primary values are different then we would have obtained a matrix $R$ with entries not in the interval $[0,1]$, but in an interval $[-c, 1+c]$, being $c>0$, where $-c$ indicates the minimum value of matrix $R, 1+c$ indicates the maximum value of matrix $R$. In such a case, we would need to transform the obtained values using a transformation function which preserves reciprocity and additive consistency, which is a function $f:[-c, 1+c] \rightarrow[0,1], f(x)=\frac{x+c}{1+2 c}$.

\section{Interval fuzzy preference relations}

Let $\tilde{a}_{1}=\left[a_{1}^{-}, a_{1}^{+}\right], \tilde{a}_{2}=\left[a_{2}^{-}, a_{2}^{+}\right], \tilde{a}=\left[a^{-}, a^{+}\right]$be three positive interval numbers, then:
(a) $\tilde{a}_{1} \oplus \tilde{a}_{2}=\left[a_{1}^{-}, a_{1}^{+}\right] \oplus\left[a_{2}^{-}, a_{2}^{+}\right]=\left[a_{1}^{-}+a_{2}^{-}, a_{1}^{+}+a_{2}^{+}\right]$;
(b) $\tilde{a}_{1} \ominus \tilde{a}_{2}=\left[a_{1}^{-}, a_{1}^{+}\right] \ominus\left[a_{2}^{-}, a_{2}^{+}\right]=\left[a_{1}^{-}-a_{2}^{+}, a_{1}^{+}-a_{2}^{-}\right]$;
(c) $\tilde{a}_{1} \otimes \tilde{a}_{2}=\left[a_{1}^{-}, a_{1}^{+}\right] \otimes\left[a_{2}^{-}, a_{2}^{+}\right]=\left[a_{1}^{-} \times a_{2}^{-}, a_{1}^{+} \times a_{2}^{+}\right]$;
(d) $\tilde{a}_{1} \varnothing \tilde{a}_{2}=\left[a_{1}^{-}, a_{1}^{+}\right] \varnothing\left[a_{2}^{-}, a_{2}^{+}\right]=\left[a_{1}^{-} / a_{2}^{+}, a_{1}^{+} / a_{2}^{-}\right]$; 
(e) $\log _{n}(\tilde{a})=\left[\log _{n} a^{-}, \log _{n} a^{+}\right]$;

(f) $\tilde{a}^{-1}=\left[1 / a^{+}, 1 / a^{-}\right]$.

Definition 3. An interval fuzzy preference $\tilde{A}=\left[\tilde{a}_{i j}\right]_{n \times n}$ is multiplicative reciprocal if and only if $\tilde{a}_{j i}=\tilde{a}_{i j}^{-1}$.

Definition 4 (Xu 2010). If a positive interval multiplicative preference relation $\tilde{A}=\left[\tilde{a}_{i j}\right]_{n \times n}$ satisfy:

$$
\tilde{a}_{i j}=\tilde{a}_{i k} \otimes \tilde{a}_{k j} \text { for all } i, k, j=1,2, \ldots, n \text {, and } i \leq k \leq j,
$$

then we call $\tilde{A}=\left(\tilde{a}_{i j}\right)$ is interval multiplicative consistent.

Note that if the interval multiplicative preference relation is consistent, for all $i, k, j=1,2, \ldots, n$, it needs satisfy $i \leq k \leq j$, otherwise, $\tilde{A}=\left[\tilde{a}_{i j}\right]_{n \times n}$ would be reduced to the crisp number judgment matrix (Saaty's reciprocal multiplicative preference relation). Further reading can refer (Xu 2010). That is Eq. (8) holds only for the upper (or lower) triangular of the preference relation.

Proposition 5. Suppose that we have a set of alternatives $X=\left\{x_{1}, x_{2}, \ldots, x_{n}\right\}$, and associated with it an interval reciprocal multiplicative preference relation $\tilde{A}=\left[\tilde{a}_{i j}\right]_{n \times n}$, with $\tilde{a}_{j i}=\tilde{a}_{i j}^{-1}$, then, the corresponding reciprocal fuzzy preference relation, $\tilde{R}=\left[\tilde{r}_{i j}\right]_{n \times n}$, associated with $\tilde{A}$ is:

$$
\tilde{r}_{i j}=g\left(\tilde{a}_{i j}\right)=\frac{1}{2}\left(1+\log _{9} \tilde{a}_{i j}\right)
$$

which verifies:

(a) $r_{i j}^{-}+r_{j i}^{+}=1, \forall i, j \in\{1,2, \ldots, n\}$;

(b) $r_{i j}^{+}+r_{j i}^{-}=1 . \forall i, j \in\{1,2, \ldots, n\}$.

Proof. By Definition 3, for $\tilde{A}=\left[\tilde{a}_{i j}\right]_{n \times n}$ being an interval reciprocal multiplicative preference relation, $\tilde{a}_{j i}=\tilde{a}_{i j}^{-1}$, that is:

Thus:

$$
\left[a_{j i}^{-}, a_{j i}^{+}\right]=\left[\frac{1}{a_{i j}^{+}}, \frac{1}{a_{i j}^{-}}\right] .
$$

$$
\begin{aligned}
& a_{j i}^{-}=\frac{1}{a_{i j}^{+}}, a_{j i}^{+}=\frac{1}{a_{i j}^{-}} ; \\
& a_{j i}^{-} a_{i j}^{+}=1, a_{j i}^{+} a_{i j}^{-}=1 .
\end{aligned}
$$

By Proposition 1 and Eq. (9) and the operational law (Eq. (6)) of interval numbers, we have:

$$
\tilde{r}_{i j}=\frac{1}{2}\left(1+\log _{9} \tilde{a}_{i j}\right)=\frac{1}{2}\left(1+\log _{9}\left[a_{i j}^{-}, a_{i j}^{+}\right]\right) .
$$

Thus:

$$
\begin{aligned}
& r_{i j}^{-}=\frac{1}{2}\left(1+\log _{9} a_{i j}^{-}\right) ; \\
& r_{i j}^{+}=\frac{1}{2}\left(1+\log _{9} a_{i j}^{+}\right) .
\end{aligned}
$$


Similarly:

Therefore:

$$
\begin{aligned}
& r_{j i}^{-}=\frac{1}{2}\left(1+\log _{9} a_{j i}^{-}\right) ; \\
& r_{j i}^{+}=\frac{1}{2}\left(1+\log _{9} a_{j i}^{+}\right) .
\end{aligned}
$$

$$
\begin{gathered}
r_{i j}^{-}+r_{j i}^{+}=\frac{1}{2}\left(1+\log _{9} a_{i j}^{-}\right)+\frac{1}{2}\left(1+\log _{9} a_{j i}^{+}\right)=1+\log _{9} a_{i j}^{-} a_{j i}^{+}=1 ; \\
r_{i j}^{+}+r_{j i}^{-}=\frac{1}{2}\left(1+\log _{9} a_{i j}^{+}\right)+\frac{1}{2}\left(1+\log _{9} a_{j i}^{-}\right)=1+\log _{9} a_{i j}^{+} a_{j i}^{-}=1, \forall i, j \in\{1,2, \ldots, n\},
\end{gathered}
$$

which completes the proof.

Proposition 6. If a reciprocal multiplicative preference relation $\tilde{A}=\left[\tilde{a}_{i j}\right]$ is multiplicative consistent, then its corresponding reciprocal fuzzy preference relation $\tilde{R}=\left[\tilde{r}_{i j}\right]$ is additive consistent, which verifies:

(a) $r_{i j}^{-}+r_{j k}^{-}+r_{k i}^{+}=\frac{3}{2}, \forall i<j<k$;

(b) $r_{i j}^{+}+r_{j k}^{+}+r_{k i}^{-}=\frac{3}{2}, \forall i<j<k$;

(c) $r_{i(i+1)}^{-}+r_{(i+1)(i+2)}^{-}+\ldots+r_{(j-1) j}^{-}+r_{j i}^{+}=\frac{j-i+1}{2}, \forall i<j$;

(d) $r_{i(i+1)}^{+}+r_{(i+1)(i+2)}^{+}+\ldots+r_{(j-1) j}^{+}+r_{j i}^{-}=\frac{j-i+1}{2}, \forall i<j$;

(e) $r_{i j_{1}}^{-}+r_{j_{1} j_{2}}^{-}+\ldots+r_{j_{t-1} j_{t}}^{-}+r_{j_{t} i}^{+}=\frac{t+1}{2}, \forall i<j_{1}<j_{2}<\ldots<j_{t}$;

(f) $r_{i j_{1}}^{+}+r_{j_{1} j_{2}}^{+}+\ldots+r_{j_{t-1} j_{t}}^{+}+r_{j_{t} i}^{-}=\frac{t+1}{2}, \forall i<j_{1}<j_{2}<\ldots<j_{t}$.

Proof. By Definition 4, for being $\tilde{A}=\left[\tilde{a}_{i j}\right]$ is multiplicative consistent, then $\tilde{a}_{i j} \otimes \tilde{a}_{j k}=\tilde{a}_{i k}$, for $\forall i<j<k$. Taking logarithm by Eq. (6) on both sides yields:

$$
\log _{9} \tilde{a}_{i j} \oplus \log _{9} \tilde{a}_{j k}=\log _{9} \tilde{a}_{i k}, \forall i<j<k .
$$

Thus:

$$
\frac{1}{2}\left(1+\log _{9} \tilde{a}_{i j}\right) \oplus \frac{1}{2}\left(1+\log _{9} \tilde{a}_{j k}\right)=\frac{1}{2}\left(1+\log _{9} \tilde{a}_{i k}\right)+\frac{1}{2} .
$$

By Eq. (9), we have:

$$
\begin{gathered}
\tilde{r}_{i j} \oplus \tilde{r}_{j k}=\tilde{r}_{i k}+\frac{1}{2} ; \\
{\left[r_{i j}^{-}, r_{i j}^{+}\right] \oplus\left[r_{j k}^{-}, r_{j k}^{+}\right]=\left[r_{i k}^{-}, r_{i k}^{+}\right]+\frac{1}{2} ;} \\
r_{i j}^{-}+r_{j k}^{-}=r_{i k}^{-}+\frac{1}{2}, r_{i j}^{+}+r_{j k}^{+}=r_{i k}^{+}+\frac{1}{2} .
\end{gathered}
$$


By Proposition 5, we have:

$$
r_{i j}^{-}+r_{j k}^{-}+r_{k i}^{+}=\frac{3}{2}, r_{i j}^{+}+r_{j k}^{+}+r_{k i}^{-}=\frac{3}{2}, \forall i<j<k .
$$

Thus the expressions (a) and (b) are established.

Let $i<j$, and $k=j-i$. The expression (c) can be rewritten as follows:

$$
r_{i(i+1)}^{-}+r_{(i+1)(i+2)}^{-}+\ldots+r_{(j-1) j}^{-}+r_{j i}^{+}=\frac{k+1}{2}, \forall i<j .
$$

Mathematical induction is used to prove this part of the proposition. The base clause is clearly true for $k=1$. The recursion clause requires it to be demonstrated that the hypothesis is true for $k=n$ :

$$
r_{i(i+1)}^{-}+r_{(i+1)(i+2)}^{-}+\ldots+r_{(i+n-1)(i+n)}^{-}+r_{(i+n) i}^{+}=\frac{n+1}{2},
$$

then it is true for $k=n+1$ :

$$
\begin{gathered}
r_{i(i+1)}^{-}+r_{(i+1)(i+2)}^{-}+\ldots+r_{(i+n-1)(i+n)}^{-}+r_{(i+n)(i+n+1)}^{-}+r_{(i+n+1) i}^{+}= \\
\left(r_{i(i+1)}^{-}+r_{(i+1)(i+2)}^{-}+\ldots+r_{(i+n-1)(i+n)}^{-}\right)+r_{(i+n)(i+n+1)}^{-}+r_{(i+n+1) i}^{+}= \\
\left(\frac{n+1}{2}-r_{(i+n) i}^{+}\right)+r_{(i+n)(i+n+1)}^{-}+r_{(i+n+1) i}^{+}= \\
\left(\frac{n+1}{2}+r_{i(i+n)}^{-}-1\right)+r_{(i+n)(i+n+1)}^{-}+r_{(i+n+1) i}^{+}= \\
\frac{n-1}{2}+\frac{3}{2}=\frac{n+2}{2}
\end{gathered}
$$

thus the expression (c) is established. Similarly, expressions (d)-(f) are established.

Proposition 7. The interval reciprocal fuzzy preference relation $\tilde{R}=\left[\tilde{r}_{i j}\right]$ is additive consistent if and only if (1) $r_{i j}^{-}-r_{i k}^{-}=r_{l j}^{-}-r_{l k}^{-} ;(2) r_{i j}^{+}-r_{i k}^{+}=r_{l j}^{+}-r_{l k}^{+}, \forall i<j<k$.

Proof. If the interval fuzzy preference relation $\tilde{R}=\left[\tilde{r}_{i j}\right]$ is additive consistent, then:

$$
\begin{gathered}
\tilde{r}_{i j} \oplus \tilde{r}_{j k}=\tilde{r}_{i k}+\frac{1}{2}, \forall i<j<k ; \\
r_{i j}^{-}+r_{j k}^{-}=r_{i k}^{-}+\frac{1}{2}, r_{i j}^{+}+r_{j k}^{+}=r_{i k}^{+}+\frac{1}{2}, \forall i<j<k ; \\
r_{i j}^{-}-r_{i k}^{-}=\frac{1}{2}-r_{j k}^{-}, r_{i j}^{+}-r_{i k}^{+}=\frac{1}{2}-r_{j k}^{+}, \forall i<j<k .
\end{gathered}
$$

Similarly:

$$
\begin{gathered}
\tilde{r}_{l j} \oplus \tilde{r}_{j k}=\tilde{r}_{l k}+\frac{1}{2}, \forall l<j<k ; \\
r_{l j}^{-}+r_{j k}^{-}=r_{l k}^{-}+\frac{1}{2}, r_{l j}^{+}+r_{l k}^{+}=r_{l k}^{+}+\frac{1}{2}, \forall l<j<k ; \\
r_{l j}^{-}-r_{l k}^{-}=\frac{1}{2}-r_{j k}^{-}, r_{l j}^{+}-r_{l k}^{+}=\frac{1}{2}-r_{l k}^{+}, \forall l<j<k,
\end{gathered}
$$


thus:

$$
r_{i j}^{-}-r_{i k}^{-}=r_{l j}^{-}-r_{l k}^{-}, r_{i j}^{+}-r_{i k}^{+}=r_{l j}^{+}-r_{l k}^{+}, \forall i, l<j<k .
$$

On the contrary:

If $r_{i j}^{-}-r_{i k}^{-}=r_{l j}^{-}-r_{l k}^{-}, r_{i j}^{+}-r_{i k}^{+}=r_{l j}^{+}-r_{l k}^{+}, \forall i<j<k$,

let $l=j$, since $r_{j j}^{-}=r_{j j}^{+}=0.5$, then

$$
r_{i j}^{-}-r_{i k}^{-}=0.5-r_{j k}^{-}, r_{i j}^{+}-r_{i k}^{+}=0.5-r_{j k}^{+}, \forall i<j<k .
$$

That is:

$$
\begin{gathered}
r_{i j}^{-}+r_{j k}^{-}=0.5+r_{i k}^{-}, r_{i j}^{+}+r_{j k}^{+}=0.5+r_{i k}^{+}, \forall i<j<k ; \\
\tilde{r}_{i j} \oplus \tilde{r}_{j k}=\tilde{r}_{i k}+\frac{1}{2}, \forall i<j<k,
\end{gathered}
$$

which completes the proof.

Proposition 7 means that for an interval fuzzy reciprocal additive consistency preference relation, the values in the upper triangular (or lower triangular) of the preference relation, the difference values of any two rows in the same column should be a constant. It will be more convenient to complete an incomplete interval fuzzy preference relation. We will use this property in the examples.

We make note that, if the primary values are different then we would have obtained a matrix $\tilde{R}$ with entries not in the interval $[0,1]$, but in an interval $[-c, 1+c]$, being $c>0$, where $-c$ indicates the minimum value of matrix $\tilde{R}, 1+c$ indicates the maximum value of matrix $R$. In such a case, we would need to transform the obtained values using a transformation function which preserves reciprocity and additive consistency, which is a function $f:[-c, 1+c] \rightarrow[0,1]$, verifying:

(a) $f(-c)=0$;

(b) $f(1+c)=1$;

(c) $f\left(x^{-}\right)+f\left(x^{+}\right)=1, \forall x \in[-c, 1+c]$;

(d) $f\left(x^{-}\right)+f\left(y^{-}\right)+f\left(z^{+}\right)=\frac{3}{2}, \forall x^{-}, y^{-}, z^{+} \in[-c, 1+c]$ such that $x^{-}+y^{-}+z^{+}=\frac{3}{2}$;

(e) $f\left(x^{+}\right)+f\left(y^{+}\right)+f\left(z^{-}\right)=\frac{3}{2}, \forall x^{+}, y^{+}, z^{-} \in[-c, 1+c]$ such that $x^{+}+y^{+}+z^{-}=\frac{3}{2}$.

The linear solution verifying (a) and (b) take the form:

$$
\begin{aligned}
& f\left(x^{-}\right)=\varphi x^{-}+\beta, \text { being } \varphi, \beta \in R ; \\
& f\left(x^{+}\right)=\varphi x^{+}+\beta, \text { being } \varphi, \beta \in R .
\end{aligned}
$$

These functions are:

$$
f\left(x^{-}\right)=\frac{1}{1+2 c} x^{-}+\frac{c}{1+2 c}=\frac{x^{-}+c}{1+2 c}
$$




$$
f\left(x^{+}\right)=\frac{1}{1+2 c} x^{+}+\frac{c}{1+2 c}=\frac{x^{+}+c}{1+2 c},
$$

which verify (c):

$$
f\left(x^{-}\right)+f\left(x^{+}\right)=\frac{x^{-}+c}{1+2 c}+\frac{x^{+}+c}{1+2 c}=\frac{\left(x^{-}+x^{+}\right)+2 c}{1+2 c}=1
$$

and when $x^{-}+y^{-}+z^{+}=\frac{3}{2}, x^{+}+y^{+}+z^{-}=\frac{3}{2}$;

$$
\begin{gathered}
f\left(x^{-}\right)+f\left(y^{-}\right)+f\left(z^{+}\right)=\frac{x^{-}+c}{1+2 c}+\frac{y^{-}+c}{1+2 c}+\frac{z^{+}+c}{1+2 c}= \\
\frac{\left(x^{-}+y^{-}+z^{+}\right)+3 c}{1+2 c}=\frac{3 / 2+3 c}{1+2 c}=\frac{3}{2} ; \\
f\left(x^{+}\right)+f\left(y^{+}\right)+f\left(z^{-}\right)=\frac{x^{+}+c}{1+2 c}+\frac{y^{+}+c}{1+2 c}+\frac{z^{-}+c}{1+2 c}= \\
\frac{\left(x^{+}+y^{+}+z^{-}\right)+3 c}{1+2 c}=\frac{3 / 2+3 c}{1+2 c}=\frac{3}{2},
\end{gathered}
$$

verify (d) and (e).

Proposition 8. Let $\tilde{R}^{(1)}, \tilde{R}^{(2)}, \ldots, \tilde{R}^{(m)}$ be the $m$ interval additive preference relations, then their weighted combination:

$$
\tilde{R}=\lambda_{1} \tilde{R}^{(1)} \oplus \lambda_{2} \tilde{R}^{(2)} \oplus \ldots \oplus \lambda_{m} \tilde{R}^{(m)}, \lambda_{l} \in[0,1], \sum_{l=1}^{m} \lambda_{l}=1,
$$

is the combined relation $\tilde{R}$, which satisfies:

$$
r_{i j}^{-}+r_{j i}^{+}=1, r_{i j}^{+}+r_{j i}^{-}=1, \forall i, j \in\{1,2, \ldots, n\} .
$$

Proof. Since $\tilde{R}^{(1)}, \tilde{R}^{(2)}, \ldots, \tilde{R}^{(m)}$ are the interval additive preference relations, it follows that:

$$
r_{i j}^{(l)-}+r_{j i}^{(l)+}=1, r_{i j}^{(l)+}+r_{j i}^{(l)-}=1, \forall i, j \in\{1,2, \ldots, n\}, l=1,2, \ldots, m .
$$

Then by Eq. (18), we have:

$$
\begin{gathered}
r_{i j}^{-}=\lambda_{1} r_{i j}^{(1)-}+\lambda_{2} r_{i j}^{(2)-}+\ldots+\lambda_{m} r_{i j}^{(m)-}, r_{i j}^{+}=\lambda_{1} r_{i j}^{(1)+}+\lambda_{2} r_{i j}^{(2)+}+\ldots+\lambda_{m} r_{i j}^{(m)+}, \forall i, j \in\{1,2, \ldots, n\} \\
r_{j i}^{-}=\lambda_{1} r_{j i}^{(1)-}+\lambda_{2} r_{j i}^{(2)-}+\ldots+\lambda_{m} r_{j i}^{(m)-}, r_{j i}^{+}=\lambda_{1} r_{j i}^{(1)+}+\lambda_{2} r_{j i}^{(2)+}+\ldots+\lambda_{m} r_{j i}^{(m)+}, \forall i, j \in\{1,2, \ldots, n\} \\
r_{i j}^{-}+r_{j i}^{+}=\lambda_{1}\left(r_{i j}^{(1)-}+r_{j i}^{(1)+}\right)+\lambda_{2}\left(r_{i j}^{(2)-}+r_{j i}^{(2)+}\right)+\ldots+\lambda_{m}\left(r_{i j}^{(m)-}+r_{j i}^{(m)+}\right)= \\
\lambda_{1}+\lambda_{2}+\ldots+\lambda_{m}=1 ; \\
r_{i j}^{+}+r_{j i}^{-}=\lambda_{1}\left(r_{i j}^{(1)+}+r_{j i}^{(1)-}\right)+\lambda_{2}\left(r_{i j}^{(2)+}+r_{j i}^{(2)-}\right)+\ldots+\lambda_{m}\left(r_{i j}^{(m)+}+r_{j i}^{(m)-}\right)= \\
\lambda_{1}+\lambda_{2}+\ldots+\lambda_{m}=1,
\end{gathered}
$$

which completes the proof. 
Proposition 9. Let $\tilde{R}^{(1)}, \tilde{R}^{(2)}, \ldots, \tilde{R}^{(m)}$ be the $m$ interval additive consistent preference relations, where $\tilde{R}^{(l)}=\left(\tilde{r}_{i j}^{(l)}\right), \tilde{r}_{i j}^{(l)} \oplus \tilde{r}_{j k}^{(l)}=\tilde{r}_{i k}^{(l)}+\frac{1}{2}, \forall i<j<k \in\{1,2, \ldots, n\}, l=1,2, \ldots, m$, then their weighted combination:

$$
\tilde{R}=\lambda_{1} \tilde{R}^{(1)} \oplus \lambda_{2} \tilde{R}^{(2)} \oplus \ldots \oplus \lambda_{m} \tilde{R}^{(m)}, \lambda_{l} \in[0,1], \sum_{l=1}^{m} \lambda_{l}=1,
$$

is the interval additive consistent preference relation, which satisfies:

$$
\tilde{r}_{i j} \oplus \tilde{r}_{j k}=\tilde{r}_{i k}+\frac{1}{2}, \forall i<j<k \text {. }
$$

Proof. Since $\tilde{R}^{(1)}, \tilde{R}^{(2)}, \ldots, \tilde{R}^{(m)}$ are interval additive consistent preference relations, which verify:

$$
\tilde{r}_{i j}^{(l)} \oplus \tilde{r}_{j k}^{(l)}=\tilde{r}_{i k}^{(l)}+\frac{1}{2}, \forall i<j<k,
$$

then by Eq. (19), we have:

$$
\begin{gathered}
\tilde{r}_{i j}=\lambda_{1} \tilde{r}_{i j}^{(1)} \oplus \lambda_{2} \tilde{r}_{i j}^{(2)} \oplus \ldots \oplus \lambda_{m} \tilde{r}_{i j}^{(m)} ; \\
\tilde{r}_{j k}=\lambda_{1} \tilde{r}_{j k}^{(1)} \oplus \lambda_{2} \tilde{r}_{j k}^{(2)} \oplus \ldots \oplus \lambda_{m} \tilde{r}_{j k}^{(m)} ; \\
\tilde{r}_{i k}=\lambda_{1} \tilde{r}_{i k}^{(1)} \oplus \lambda_{2} \tilde{r}_{i k}^{(2)} \oplus \ldots \oplus \lambda_{m} \tilde{r}_{i k}^{(m)} ; \\
\tilde{r}_{i j} \oplus \tilde{r}_{j k}=\lambda_{1}\left(\tilde{r}_{i j}^{(1)} \oplus \tilde{r}_{j k}^{(1)}\right) \oplus \lambda_{2}\left(\tilde{r}_{i j}^{(2)} \oplus \tilde{r}_{j k}^{(2)}\right) \oplus \ldots \oplus \lambda_{m}\left(\tilde{r}_{i j}^{(m)} \oplus \tilde{r}_{j k}^{(m)}\right)= \\
\lambda_{1}\left(r_{i k}^{(1)}+\frac{1}{2}\right) \oplus \lambda_{2}\left(r_{i k}^{(2)}+\frac{1}{2}\right) \oplus \ldots \oplus \lambda_{m}\left(r_{i k}^{(m)}+\frac{1}{2}\right)= \\
\lambda_{1} \tilde{r}_{i k}^{(1)} \oplus \lambda_{2} \tilde{r}_{i k}^{(2)} \oplus \ldots \oplus \lambda_{m} \tilde{r}_{i k}^{(m)}+\frac{\left(\lambda_{1}+\lambda_{2}+\ldots+\lambda_{m}\right)}{2}=\tilde{r}_{i k}+\frac{1}{2},
\end{gathered}
$$

which completes the proof.

\section{Procedures for incomplete interval fuzzy preference relations}

A complete preference relation requires $n(n-1) / 2$ judgments in its entire top triangular portion. Sometimes, however, a decision maker (DM) may develop a preference relation with incomplete information due to lack of time and knowledge, and the DM's limited experience related with the problem domain. In the following, we present some concepts of incomplete interval additive preference relation.

Definition 5. Let $\tilde{R}=\left[\tilde{r}_{i j}\right]$ be an interval additive preference relation, then $\tilde{R}$ is called an incomplete interval additive preference relation, if some of its elements cannot be given by the decision maker, which we denote by the known variable " $x$ ", and others can be provided by the decision maker, if which satisfy (1) $\tilde{r}_{i j}=1-\tilde{r}_{j i}, \tilde{r}_{i i}=[0.5,0.5]$, we call it incomplete interval additive preference relation. 
Definition 6. Let $\tilde{R}=\left[\tilde{r}_{i j}\right]$ be an incomplete interval additive preference relation, if each known element can be obtained by its adjoining known elements, then $\tilde{R}$ is called acceptable, otherwise, $\tilde{R}$ is unacceptable.

Proposition 10. Let $\tilde{R}=\left[\tilde{r}_{i j}\right]$ be an incomplete interval additive preference relation, if $\tilde{R}$ is acceptable, then there exists at least one known element (except diagonal element) in each line or each column of $\tilde{R}$, i.e. there exist at least $(n-1)$ judgments provided by the decision maker.

Definition 7. Let $\tilde{R}=\left[\tilde{r}_{i j}\right]$ be an incomplete interval additive preference relation, if:

$$
\tilde{r}_{i j} \oplus \tilde{r}_{j k}=\tilde{r}_{i k} \oplus 0.5 \text {, for } i<j<k,
$$

then $\tilde{R}$ is called an additive consistent incomplete interval preference relation.

In the following, based on the acceptable incomplete interval additive preference relation with the least judgments (i.e. $n-1$ judgments), we shall develop a simple and practical method for constructing a complete additive consistent interval preference relation, and also rank alternatives as follows:

Step 1. For a decision problem, let $X=\left\{x_{1}, x_{2}, \ldots, x_{n}\right\}$ be a discrete set of alternatives. The decision maker compares each pair of alternatives by the interval additive preference relation, and construct an acceptable incomplete interval additive preference relation $\tilde{R}=\left[\tilde{r}_{i j}\right]_{n \times n}$ (if the decision maker provides the acceptable incomplete interval multiplicative preference relation $\tilde{A}=\left[\tilde{a}_{i j}\right]_{n \times n}$, then we can transfer $\tilde{A}=\left[\tilde{a}_{i j}\right]_{n \times n}$ to the corresponding incomplete interval additive preference relation $\tilde{R}=\left[\tilde{r}_{i j}\right]_{n \times n}$ by Proposition 5 ), with only $n-1$ judgments $\tilde{r}_{i_{1} j_{1}}, \tilde{r}_{i_{2} j_{2}}, \ldots, \tilde{r}_{i_{n-1} j_{n-1}}$.

Step 2. Utilize Proposition 6 or Proposition 7 to determine all the unknown elements in $\tilde{R}$, and thus get an interval additive consistent preference relation $\overline{\tilde{R}}=\left[\overline{\tilde{r}}_{i j}\right]_{n \times n}$. If this preference relation contains any values which are not in the interval $[0,1]$, but in the interval $[-c, 1+c]$, then transformation functions are required to preserve the reciprocity and additive transitivity. The transformation functions are given by Eqs (13)-(17).

Step 3. Utilize the interval normalizing rank aggregation method:

$$
\tilde{w}_{i}=\frac{\sum_{j=1}^{n} \overline{\tilde{r}}_{i j}}{\sum_{i=1}^{n} \sum_{j=1}^{n} \overline{\tilde{r}}_{i j}}, i=1,2, \ldots, n .
$$

And then acquire the average degree $\tilde{w}_{i}$ of the $i$ th alternative over all other alternatives.

Step 4. Utilize the following formula:

$$
p\left(\tilde{w}_{i} \geq \tilde{w}_{j}\right)=\min \left\{\max \left(\frac{\tilde{w}_{i}^{+}-\tilde{w}_{j}^{-}}{\tilde{w}_{i}^{+}-\tilde{w}_{i}^{-}+\tilde{w}_{j}^{+}-\tilde{w}_{j}^{-}}, 0\right), 1\right\},
$$

to compare each $\tilde{w}_{i}$ with all $\tilde{w}_{j}(j=1,2, \ldots, n)$, we get the possibility degree $p_{i j}=p\left(\tilde{w}_{i} \geq \tilde{w}_{j}\right)$, and then construct a complementary matrix as $P=\left[p_{i j}\right]_{n \times n}$, where:

$$
p_{i j} \geq 0, p_{i j}+p_{j i}=1, p_{i i}=\frac{1}{2}, i, j=1,2, \ldots, n .
$$


Step 5. Utilize the normalizing rank aggregation method (Xu et al. 2009) given by:

$$
\varpi_{i}=\frac{\sum_{j=1}^{n} p_{i j}}{n^{2} / 2}, i=1,2, \ldots, n,
$$

to derive the priority vector $\varpi=\left(\varpi_{1}, \varpi_{2}, \ldots, \varpi_{n}\right)^{T}$ of the complementary matrix $P$. Then we rank the alternatives $x_{i}(i=1,2, \ldots, n\}$ in descending order in accordance with the values of $\varpi_{i}(i=1,2, \ldots ., n)$.

Step 6. End.

In real world, however, many decision making processes take place in multi-person settings because the increasing complexity and uncertainty of the socio-economic environment makes it less and less possible for single decision maker to consider all relevant aspects of a decision making problem. In the following, we consider the hierarchy problem with group decision making.

For a hierarchy decision problem, let $X=\left\{x_{1}, x_{2}, \ldots, x_{n}\right\}$ be a discrete set of alternatives, let $C=\left\{c_{1}, c_{2}, \ldots, c_{m}\right\}$ be a set of criteria, let $D=\left\{d_{1}, d_{2}, \ldots, d_{l}\right\}$ be a set of decision makers, $\lambda=\left(\lambda_{1}, \lambda_{2}, \ldots, \lambda_{l}\right)^{T}$ be the weight vector of decision makers, where $\lambda_{k} \geq 0, k=1,2, \ldots, l$, $\sum_{k=1}^{l} \lambda_{k}=1$. The procedure for the incomplete interval AHP is described as follows:

Part A. Determine the priority weights of criteria.

Step 1. The decision maker $d_{k} \in D$ compares each pair of criteria by the interval additive preference relation, and construct an acceptable incomplete interval additive preference relation $\tilde{R}^{(k)}=\left[\tilde{r}_{i j}^{(k)}\right]_{m \times m}$ (if the decision maker provides the acceptable incomplete interval multiplicative preference relation $\tilde{A}^{(k)}=\left[\tilde{a}_{i j}^{(k)}\right]_{m \times m}$, then we can transfer $\tilde{A}^{(k)}=\left[\tilde{a}_{i j}^{(k)}\right]_{m \times m}$ to the corresponding incomplete interval additive preference relation $\tilde{R}^{(k)}=\left[\tilde{r}_{i j}^{(k)}\right]_{m \times m}$ by Proposition 5), with only $m$-1 judgments $\tilde{r}_{i_{1} j_{1}}^{(k)}, \tilde{r}_{i_{2} j_{2}}^{(k)}, \ldots, \tilde{r}_{i_{m-1} j_{m-1}}^{(k)}$.

Step 2. Utilize Proposition 6 or Proposition 7 to determine all the unknown elements in $\tilde{R}^{(k)}$, and thus get an interval additive consistent preference relation $\overline{\tilde{R}}^{(k)}=\left[\overline{\tilde{r}}_{i j}^{(k)}\right]_{m \times m}$. If this preference relation contains any values which are not in the interval $[0,1]$, but in the interval $[-c, 1+c]$, then a transformation functions are required to preserve the reciprocity and additive transitivity. The transformation functions are given by Eqs (13)-(17).

Step 3. Utilize the interval additive weighted averaging operator:

$$
\overline{\tilde{r}}_{i j}=\lambda_{1} \overline{\tilde{r}}_{i j}^{(1)} \oplus \lambda_{2} \overline{\tilde{r}}_{i j}^{(2)} \oplus \ldots \oplus \lambda_{l} \overline{\tilde{r}}_{i j}^{(l)},
$$

to fuse all the interval additive consistent preference relations $\overline{\tilde{R}}^{(k)}(k=1,2, \ldots, l)$ into a collective complete interval additive preference relation $\overline{\tilde{R}}=\left[\overline{\tilde{r}}_{i j}\right]_{m \times m}$.

Step 4. Utilize the interval normalizing rank aggregation method:

$$
\tilde{w}_{i}=\frac{\sum_{j=1}^{m} \overline{\tilde{r}}_{i j}}{\sum_{i=1}^{m} \sum_{j=1}^{m} \overline{\tilde{r}}_{i j}}, i=1,2, \ldots ., m,
$$

to determine the priority weight of the criteria. 
Part B. Determine the priority ratings for each alternative with respect to each criterion.

Step 1. The decision maker $d_{k} \in D$ compares each pair of alternative with respect to each criteria $i(i=1,2, \ldots, m)$ by the incomplete interval additive preference relation, and construct an acceptable incomplete interval additive preference relation ${ }_{i} \tilde{B}^{(k)}=\left[{ }_{i} \tilde{b}_{u v}^{(k)}\right]_{n \times n}$, (if the DM provides the acceptable incomplete interval multiplicative preference relation ${ }_{i} \tilde{A}^{(k)}=\left[\tilde{a}_{i j}^{(k)}\right]_{n \times n}$, then we can transfer ${ }_{i} \tilde{A}^{(k)}=\left[\tilde{a}_{i j}^{(k)}\right]_{n \times n}$ to the corresponding incomplete interval additive preference relation ${ }_{i} \tilde{B}^{(k)}=\left[{ }_{i} \tilde{b}_{u v}^{(k)}\right]_{n \times n}$ by Proposition 5), with only $n-1$ judgments ${ }_{i} \tilde{b}_{u_{1} v_{1},{ }_{i}}^{(k)} \tilde{b}_{u_{2} v_{2}}^{(k)}, \ldots,{ }_{i} \tilde{b}_{u_{n-1} v_{n-1}}^{(k)}$.

Step 2. Utilize Proposition 6 or Proposition 7 to determine all the unknown elements in ${ }_{i} \tilde{B}^{(k)}$, and thus get an interval additive consistent preference relation ${ }_{i} \overline{\tilde{B}}^{(k)}=\left[{ }_{i} \overline{\tilde{b}}_{u v}^{(k)}\right]_{n \times n}$. If this preference relation contains any values which are not in the interval $[0,1]$, but in the interval $[-c, 1+c]$, then transformation functions are required to preserve the reciprocity and additive transitivity. The transformation functions are given by Eqs (13)-(17).

Step 3. Utilize the interval additive weighted averaging operator:

$$
{ }_{i} \overline{\tilde{b}}_{u v}=\lambda_{1 i} \overline{\tilde{b}}_{u v}^{(1)} \oplus \lambda_{2 i} \overline{\tilde{b}}_{u v}^{(3)} \oplus \ldots \oplus \lambda_{l i} \overline{\tilde{b}}_{u v}^{(l)},
$$

to fuse all DMs' interval additive consistent preference relations ${ }_{i} \overline{\tilde{B}}^{(k)}(k=1,2, \ldots, l)$ into a collective complete interval additive preference relation ${ }_{i} \overline{\tilde{B}}=\left[{ }_{i} \overline{\tilde{b}}_{u v}\right]_{n \times n}$ with respect to each criteria.

Step 4. Utilize the interval normalizing rank aggregation method:

$$
\overline{\tilde{b}}_{u}=\frac{\sum_{v=1}^{n} \overline{\tilde{b}}_{u v}}{\sum_{v=1}^{n} \sum_{u=1}^{n} \overline{\tilde{b}}_{u v}}, i=1,2, \ldots ., m
$$

to determine the priority ratings for each alternative with respect to each criteria $i(i=1,2, \ldots ., m)$.

Part C. Obtaining the priority weight for each alternative.

Step 1. Multiplying the priority weights of each criteria $i$ by the priority ratings of each alternative, that is:

$$
\tilde{Z}_{u}=\tilde{w}_{1} \otimes_{1} \overline{\tilde{b}}_{u} \oplus \tilde{w}_{2} \otimes_{2} \overline{\tilde{b}}_{u} \oplus \ldots \oplus \tilde{w}_{m} \otimes_{m} \overline{\tilde{b}}_{u}, u=1,2, \ldots, n .
$$

Thus, we get the overall preference ratings for each alternative.

Step 2. Utilize the following formula:

$$
p\left(\tilde{Z}_{u} \geq \tilde{Z}_{v}\right)=\min \left\{\max \left(\frac{\tilde{z}_{u}^{+}-\tilde{z}_{v}^{-}}{\tilde{z}_{u}^{+}-\tilde{z}_{u}^{-}+\tilde{z}_{v}^{+}-\tilde{z}_{v}^{-}}, 0\right), 1\right\},
$$

to compare each $\tilde{Z}_{u}$ with all $\tilde{Z}_{v}(v=1,2, \ldots, n)$, we get the possibility degree $p_{u v}=p\left(\tilde{Z}_{u} \geq \tilde{Z}_{v}\right)$, and then construct a complementary matrix as $P=\left[p_{u v}\right]_{n \times n}$, where:

$$
p_{u v} \geq 0, p_{u v}+p_{v u}=1, p_{u u}=\frac{1}{2}, u, v=1,2, \ldots, n
$$


Step 3. Utilize the normalizing rank aggregation method given by:

$$
\varpi_{u}=\frac{\sum_{v=1}^{n} p_{u v}}{n^{2} / 2}, u=1,2, \ldots, n,
$$

to derive the priority vector $\varpi=\left(\varpi_{1}, \varpi_{2}, \ldots, \varpi_{n}\right)^{T}$ of the complementary matrix $P$. Then we rank the alternatives $x_{u}(u=1,2, \ldots, n)$ in descending order in accordance with the values of $\varpi_{i}(u=1,2, \ldots, n)$.

Step 4. End.

\section{Numerical examples}

In this section, we present an example and a simple case study to illustrate the applications of the proposed method to construct the consistent interval additive preference relation. In the first example, we show how an incomplete interval additive preference relation is constructed. The case study is a group decision making problem with a hierarchical structure and incomplete interval additive preference relations. It involves evaluating and selecting a supplier in supply chain management.

Example 1. In this example, a decision-making problem involves the evaluation of seven schools $x_{i}(i=1,2, \ldots, 7)$ of a university. The decision maker compares these seven schools (alternatives) by the interval additive preference relation and provides his/her judgment as follows:

$$
\tilde{r}_{31}=[0.4,0.7], \tilde{r}_{32}=[0.1,0.3], \tilde{r}_{34}=[0.3,0.4], \tilde{r}_{35}=[0.4,0.7], \tilde{r}_{36}=[0.3,0.8], \tilde{r}_{37}=[0.4,0.9] .
$$

Step 1. By Definition 5 and the above information provided by the decision maker, we get the acceptable incomplete interval fuzzy preference relation, where " $x$ " denotes the unknown variable:

$$
\tilde{R}=\left[\begin{array}{ccccccc}
{[0.5,0.5]} & x & x & x & x & x & x \\
x & {[0.5,0.5]} & & x & x & x & x \\
{[0.4,0.7]} & {[0.1,0.3]} & {[0.5,0.5]} & {[0.3,0.4]} & {[0.4,0.7]} & {[0.3,0.8]} & {[0.4,0.9]} \\
x & x & x & {[0.5,0.5]} & x & x & x \\
x & x & x & x & {[0.5,0.5]} & x & x \\
x & x & x & x & x & {[0.5,0.5]} & x \\
x & x & x & x & x & x & {[0.5,0.5]}
\end{array}\right] .
$$

Step 2. Utilize Proposition 6 and Proposition 7 to determine all the unknown elements in $\tilde{R}$, the entire calculation is as follows:

$$
\begin{aligned}
& r_{13}^{-}=1-r_{31}^{+}=0.3, r_{13}^{+}=1-r_{31}^{-}=0.6, r_{23}^{-}=1-r_{32}^{+}=0.7, r_{23}^{+}=1-r_{32}^{-}=0.9, \\
& r_{12}^{-}=\frac{3}{2}-r_{23}^{-}-r_{31}^{+}=0.1, r_{12}^{+}=\frac{3}{2}-r_{23}^{+}-r_{31}^{-}=0.2, r_{21}^{-}=1-r_{12}^{+}=0.8, r_{21}^{+}=1-r_{12}^{-}=0.9, \\
& r_{23}^{-}-r_{33}^{-}=r_{24}^{-}-r_{34}^{-} \Rightarrow r_{24}^{-}=r_{23}^{-}-r_{33}^{-}+r_{34}^{-}=0.5, r_{24}^{+}=r_{23}^{+}-r_{33}^{+}+r_{34}^{+}=0.8,
\end{aligned}
$$




$$
\begin{aligned}
& r_{25}^{-}=r_{23}^{-}-r_{33}^{-}+r_{35}^{-}=0.6, r_{25}^{+}=r_{23}^{+}-r_{33}^{+}+r_{35}^{+}=1.1, r_{26}^{-}=r_{23}^{-}-r_{33}^{-}+r_{36}^{-}=0.5 \text {, } \\
& r_{26}^{+}=r_{23}^{+}-r_{33}^{+}+r_{36}^{+}=1.2, r_{27}^{-}=r_{23}^{-}-r_{33}^{-}+r_{37}^{-}=0.6, r_{27}^{+}=r_{23}^{+}-r_{33}^{+}+r_{37}^{+}=1.3 \text {, } \\
& r_{14}^{-}=r_{13}^{-}-r_{33}^{-}+r_{34}^{-}=0.1, r_{14}^{+}=r_{13}^{+}-r_{33}^{+}+r_{34}^{+}=0.5, r_{15}^{-}=r_{13}^{-}-r_{33}^{-}+r_{35}^{-}=0.2 \text {, } \\
& r_{15}^{+}=r_{13}^{+}-r_{33}^{+}+r_{35}^{+}=0.8, r_{16}^{-}=r_{13}^{-}-r_{33}^{-}+r_{36}^{-}=0.1, r_{16}^{+}=r_{13}^{+}-r_{33}^{+}+r_{36}^{+}=0.9 \text {, } \\
& r_{17}^{-}=r_{13}^{-}-r_{33}^{-}+r_{37}^{-}=0.2, r_{17}^{+}=r_{13}^{+}-r_{33}^{+}+r_{37}^{+}=1, r_{45}^{-}=r_{35}^{-}-r_{34}^{-}+r_{44}^{-}=0.6 \text {, } \\
& r_{45}^{+}=r_{35}^{+}-r_{34}^{+}+r_{44}^{+}=0.8, r_{46}^{-}=r_{36}^{-}-r_{34}^{-}+r_{44}^{-}=0.5, r_{46}^{+}=r_{36}^{+}-r_{34}^{+}+r_{44}^{+}=0.9 \text {, } \\
& r_{47}^{-}=r_{37}^{-}-r_{34}^{-}+r_{44}^{-}=0.6, r_{47}^{+}=r_{37}^{+}-r_{34}^{+}+r_{44}^{+}=1, r_{56}^{-}=r_{36}^{-}-r_{35}^{-}+r_{55}^{-}=0.4 \text {, } \\
& r_{56}^{+}=r_{36}^{+}-r_{35}^{+}+r_{55}^{+}=0.6, r_{57}^{-}=r_{37}^{-}-r_{35}^{-}+r_{55}^{-}=0.5, r_{57}^{+}=r_{37}^{+}-r_{35}^{+}+r_{55}^{+}=0.7 \text {, } \\
& r_{67}^{-}=r_{37}^{-}-r_{36}^{-}+r_{66}^{-}=0.6, r_{67}^{+}=r_{37}^{+}-r_{36}^{+}+r_{66}^{+}=0.6, r_{41}^{-}=1-r_{14}^{+}=0.5 \text {, } \\
& r_{41}^{+}=1-r_{14}^{-}=0.9, r_{42}^{-}=1-r_{24}^{+}=0.2, r_{42}^{+}=1-r_{24}^{-}=0.5, r_{43}^{-}=1-r_{34}^{+}=0.6 \text {, } \\
& r_{43}^{+}=1-r_{34}^{-}=0.7, r_{51}^{-}=1-r_{15}^{+}=0.2, r_{51}^{+}=1-r_{15}^{-}=0.8, r_{52}^{-}=1-r_{25}^{+}=-0.1 \text {, } \\
& r_{52}^{+}=1-r_{25}^{-}=0.4, r_{53}^{-}=1-r_{35}^{+}=0.3, r_{53}^{+}=1-r_{35}^{-}=0.6, r_{54}^{-}=1-r_{45}^{+}=0.2 \text {, } \\
& r_{54}^{+}=1-r_{45}^{-}=0.4, r_{61}^{-}=1-r_{16}^{+}=0.1, r_{61}^{+}=1-r_{16}^{-}=0.8, r_{62}^{-}=1-r_{26}^{+}=-0.2 \text {, } \\
& r_{62}^{+}=1-r_{26}^{-}=0.5, r_{63}^{-}=1-r_{36}^{+}=0.2, r_{63}^{+}=1-r_{36}^{-}=0.7, r_{64}^{-}=1-r_{46}^{+}=0.1 \text {, } \\
& r_{64}^{+}=1-r_{46}^{-}=0.5, r_{65}^{-}=1-r_{56}^{+}=0.4, r_{65}^{+}=1-r_{56}^{-}=0.6, r_{71}^{-}=1-r_{17}^{+}=0 \text {, } \\
& r_{71}^{+}=1-r_{17}^{-}=0.7, r_{72}^{-}=1-r_{27}^{+}=-0.3, r_{72}^{+}=1-r_{27}^{-}=0.4, r_{73}^{-}=1-r_{37}^{+}=0.1 \text {, } \\
& r_{73}^{+}=1-r_{37}^{-}=0.6, r_{74}^{-}=1-r_{47}^{+}=0, r_{74}^{+}=1-r_{47}^{-}=0.4, r_{75}^{-}=1-r_{57}^{+}=0.3 \text {, } \\
& r_{75}^{+}=1-r_{57}^{-}=0.5, r_{76}^{-}=1-r_{67}^{+}=0.4, r_{76}^{+}=1-r_{67}^{-}=0.4 \text {; }
\end{aligned}
$$$$
\overline{\tilde{R}}=\left[\begin{array}{ccccccc}
{[0.5,0.5]} & {[0.1,0.2]} & {[0.3,0.6]} & {[0.1,0.5]} & {[0.2,0.8]} & {[0.2,0.9]} & {[0.3,1]} \\
{[0.8,0.9]} & {[0.5,0.5]} & {[0.7,0.9]} & {[0.5,0.8]} & {[0.6,1.1]} & {[0.5,1.2]} & {[0.6,1.3]} \\
{[0.4,0.7]} & {[0.1,0.3]} & {[0.5,0.5]} & {[0.3,0.4]} & {[0.4,0.7]} & {[0.3,0.8]} & {[0.4,0.9]} \\
{[0.5,0.9]} & {[0.2,0.5]} & {[0.6,0.7]} & {[0.5,0.5]} & {[0.6,0.8]} & {[0.5,0.9]} & {[0.6,1]} \\
{[0.2,0.8]} & {[-0.1,0.4]} & {[0.3,0.6]} & {[0.2,0.4]} & {[0.5,0.5]} & {[0.4,0.6]} & {[0.5,0.7]} \\
{[0.1,0.8]} & {[-0.2,0.5]} & {[0.2,0.7]} & {[0.1,0.5]} & {[0.4,0.6]} & {[0.5,0.5]} & {[0.6,0.6]} \\
{[0,0.7]} & {[-0.3,0.4]} & {[0.1,0.6]} & {[0,0.4]} & {[0.3,0.5]} & {[0.4,0.4]} & {[0.5,0.5]}
\end{array}\right] .
$$ 
The preference relation contains the values that are not included in the interval [0,1]; thus the transformation functions Eqs (13)-(17) are applied, we have:

$$
\ddot{\tilde{R}}=\left[\begin{array}{cccccccc}
{[0.5,0.5]} & {[0.25,0.31]} & {[0.38,0.56]} & {[0.25,0.5]} & {[0.31,0.69]} & {[0.31,0.75]} & {[0.38,0.81]} \\
{[0.69,0.75]} & {[0.5,0.5]} & {[0.63,0.75]} & {[0.5,0.69]} & {[0.56,0.88]} & {[0.5,0.94]} & {[0.56,1]} \\
{[0.44,0.63]} & {[0.25,0.38]} & {[0.5,0.5]} & {[0.38,0.44]} & {[0.44,0.63]} & {[0.38,0.69]} & {[0.44,0.75]} \\
{[0.5,0.75]} & {[0.31,0.5]} & {[0.56,0.63]} & {[0.5,0.5]} & {[0.56,0.69]} & {[0.5,0.75]} & {[0.56,0.81]} \\
{[0.31,0.69]} & {[0.13,0.44]} & {[0.38,0.56]} & {[0.31,0.44]} & {[0.5,0.5]} & {[0.44,0.56]} & {[0.5,0.63]} \\
{[0.25,0.69]} & {[0.06,0.5]} & {[0.31,0.63]} & {[0.25,0.5]} & {[0.44,0.56]} & {[0.5,0.5]} & {[0.56,0.56]} \\
{[0.19,0.63]} & {[0,0.44]} & {[0.25,0.56]} & {[0.19,0.44]} & {[0.3,0.5]} & {[0.44,0.44]} & {[0.5,0.5]}
\end{array}\right] .
$$

Step 3. Utilize the interval normalizing averaging operator (Eq. (20)) to fuse all the preference degrees $\ddot{\tilde{r}}_{i j}(j=1,2, \ldots, 7)$ in the $i$ th line of the $\ddot{\tilde{R}}$, and then get the averaged importance degree $\tilde{w}_{i}$ of the $i$ th alternative over all the other alternatives:

$$
\begin{gathered}
\tilde{w}_{1}=[0.081,0.212], \tilde{w}_{2}=[0.133,0.283], \tilde{w}_{3}=[0.096,0.207], \tilde{w}_{4}=[0.118,0.238], \\
\tilde{w}_{5}=[0.087,0.197], \tilde{w}_{6}=[0.08,0.203], \tilde{w}_{7}=[0.063,0.181] .
\end{gathered}
$$

Step 4. By Eq. (21), we construct the possibility degree matrix:

$$
P=\left[\begin{array}{ccccccc}
0.5 & 0.279 & 0.479 & 0.373 & 0.519 & 0.519 & 0.598 \\
0.721 & 0.5 & 0.719 & 0.612 & 0.757 & 0.746 & 0.823 \\
0.521 & 0.281 & 0.5 & 0.384 & 0.544 & 0.542 & 0.629 \\
0.627 & 0.388 & 0.616 & 0.5 & 0.659 & 0.651 & 0.737 \\
0.481 & 0.243 & 0.456 & 0.341 & 0.5 & 0.501 & 0.587 \\
0.481 & 0.254 & 0.458 & 0.349 & 0.499 & 0.5 & 0.581 \\
0.402 & 0.177 & 0.371 & 0.263 & 0.414 & 0.419 & 0.5
\end{array}\right]
$$

Step 5. By Eq. (22), we get:

$$
\begin{gathered}
\varpi_{1}=0.1334, \varpi_{2}=0.1991, \varpi_{3}=0.1388, \varpi_{4}=0.1705, \varpi_{5}=0.1268, \varpi_{6}=0.1275, \\
\varpi_{7}=0.1039
\end{gathered}
$$

and thus $\varpi_{2}>\varpi_{4}>\varpi_{3}>\varpi_{1}>\varpi_{6}>\varpi_{5}>\varpi_{7}$, by which we rank the schools:

$$
x_{2} \succ x_{4} \succ x_{3} \succ x_{1} \succ x_{6} \succ x_{5} \succ x_{7}
$$

and hence $x_{2}$ is the best school. 
Example 2. A high-technology manufacturing company desires to select a suitable material supplier to purchase the key components of new products. After preliminary screening, four candidates $\left(x_{1}, x_{2}, x_{3}, x_{4}\right)$ remain for further evaluation. A committee of three decision-makers, $d_{1}, d_{2}, d_{3}$ (whose weight vector is $\lambda=(1 / 3,1 / 3,1 / 3)^{T}$ ) has been formed to select the most suitable supplier (adapted from (Chen et al. 2006)). Five benefit criteria are considered: (1) profitability of supplier $\left(c_{1}\right) ;(2)$ relationship closeness $\left(c_{2}\right) ;(3)$ technological capability $\left(c_{3}\right) ;(4)$ conformance quality $\left(c_{4}\right) ;(5)$ conflict resolution $\left(c_{5}\right)$.

The hierarchy structure of this decision problem is shown in Figure 1. The three DMs reveal their preference relations for the criteria and candidates independently and anonymously. The DMs' incomplete interval additive preference relations are shown below.

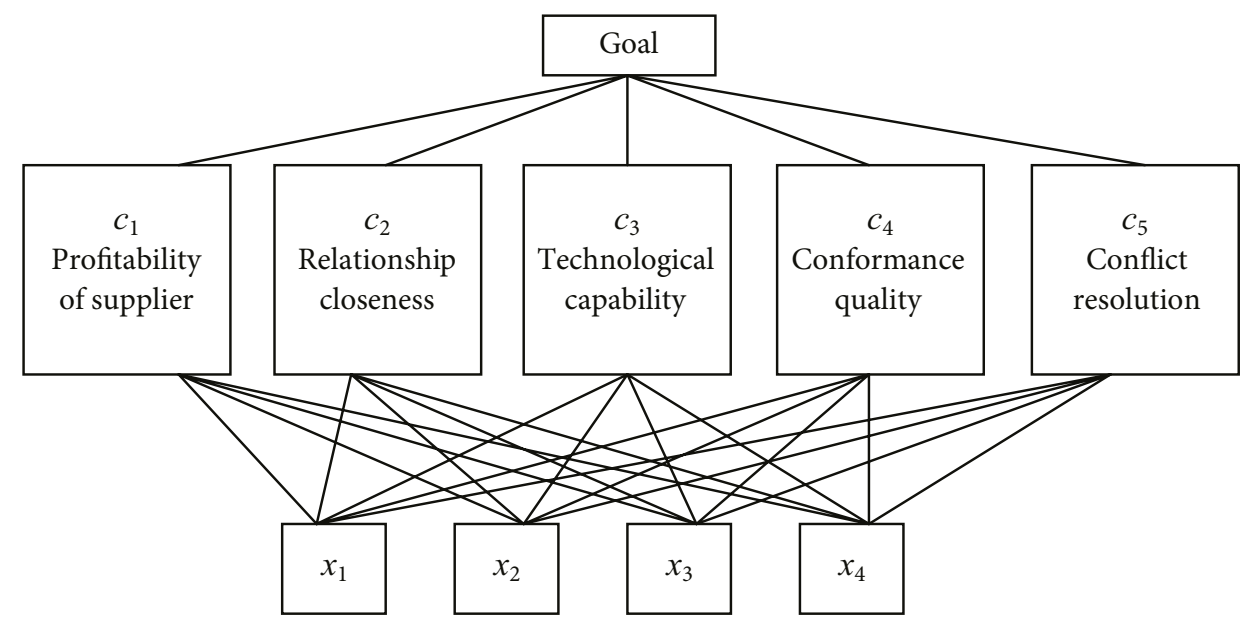

Fig. 1. Hierarchical structure of decision problem

Incomplete interval additive preference relations for five criteria by the decision makers $1,2,3$ :

$$
\begin{array}{r}
\tilde{R}^{(1)}=\left[\begin{array}{ccccc}
{[0.5,0.5]} & x & {[0.2,0.5]} & x & x \\
x & {[0.5,0.5]} & {[0.3,0.5]} & x & x \\
x & x & {[0.5,0.5]} & x & x \\
x & x & {[0.4,0.6]} & {[0.5,0.5]} & x \\
x & x & {[0.6,0.7]} & x & {[0.5,0.5]}
\end{array}\right] ; \\
\tilde{R}^{(2)}=\left[\begin{array}{ccccc}
{[0.5,0.5]} & {[0.1,0.3]} & {[0.3,0.6]} & {[0.2,0.8]} & {[0.1,0.7]} \\
x & {[0.5,0.5]} & x & x & x \\
x & x & {[0.5,0.5]} & x & x \\
x & x & x & {[0.5,0.5]} & x \\
x & x & x & x & {[0.5,0.5]}
\end{array}\right] ;
\end{array}
$$




$$
\tilde{R}^{(3)}=\left[\begin{array}{ccccc}
{[0.5,0.5]} & {[0.3,0.4]} & x & x & x \\
x & {[0.5,0.5]} & {[0.5,0.6]} & x & x \\
x & x & {[0.5,0.5]} & {[0.2,0.3]} & x \\
x & x & x & {[0.5,0.5]} & {[0.7,0.8]} \\
x & x & x & x & {[0.5,0.5]}
\end{array}\right] .
$$

Incomplete interval additive preference relation for the four candidates with respect to the profitability of supplier criterion:

$$
\begin{aligned}
& { }_{1} \tilde{B}^{(1)}=\left[\begin{array}{cccc}
{[0.5,0.5]} & {[0.8,0.9]} & {[0.5,0.8]} & {[0.4,0.8]} \\
x & {[0.5,0.5]} & x & x \\
x & x & {[0.5,0.5]} & x \\
x & x & x & {[0.5,0.5]}
\end{array}\right] ; \\
& { }_{1} \tilde{B}^{(2)}=\left[\begin{array}{cccc}
{[0.5,0.5]} & x & {[0.4,0.5]} & x \\
x & {[0.5,0.5]} & {[0.3,0.4]} & x \\
x & x & {[0.5,0.5]} & x \\
x & x & {[0.2,0.3]} & {[0.5,0.5]}
\end{array}\right] \text {; } \\
& { }_{1} \tilde{B}^{(3)}=\left[\begin{array}{cccc}
{[0.5,0.5]} & x & x & x \\
x & {[0.5,0.5]} & x & x \\
{[0.3,0.5]} & {[0.7,0.8]} & {[0.5,0.5]} & {[0.5,0.7]} \\
x & x & x & {[0.5,0.5]}
\end{array}\right] \text {. }
\end{aligned}
$$

Incomplete interval additive preference relation for the four candidates with respect to the relationship closeness criterion:

$$
\begin{aligned}
{ }_{2} \tilde{B}^{(1)} & =\left[\begin{array}{cccc}
{[0.5,0.5]} & {[0.6,0.7]} & x & x \\
x & {[0.5,0.5]} & {[0.3,0.4]} & x \\
x & x & {[0.5,0.5]} & {[0.4,0.6]} \\
x & x & x & {[0.5,0.5]}
\end{array}\right] ; \\
{ }_{2} \tilde{B}^{(2)} & =\left[\begin{array}{cccc}
{[0.5,0.5]} & x & x & {[0.7,1]} \\
x & {[0.5,0.5]} & x & {[0.3,0.5]} \\
x & x & {[0.5,0.5]} & {[0.7,0.8]} \\
x & x & x & {[0.5,0.5]}
\end{array}\right] ; \\
{ }_{2} \tilde{B}^{(3)} & =\left[\begin{array}{cccc}
{[0.5,0.5]} & x & x & x \\
x & {[0.5,0.5]} & x & x \\
{[0.2,0.3]} & {[0.6,0.7]} & {[0.5,0.5]} & {[0.6,0.7]} \\
x & x & x & {[0.5,0.5]}
\end{array}\right] .
\end{aligned}
$$


Incomplete interval additive preference relation for the four candidates with respect to the technological capability criterion:

$$
\begin{aligned}
{ }_{3} \tilde{B}^{(1)} & =\left[\begin{array}{cccc}
{[0.5,0.5]} & {[0.6,0.7]} & x & x \\
x & {[0.5,0.5]} & x & x \\
x & {[0.7,0.8]} & {[0.5,0.5]} & x \\
x & {[0.3,0.4]} & x & {[0.5,0.5]}
\end{array}\right] ; \\
{ }_{3} \tilde{B}^{(2)} & =\left[\begin{array}{cccc}
{[0.5,0.5]} & {[0.5,0.7]} & x & x \\
x & {[0.5,0.5]} & {[0.2,0.3]} & x \\
x & x & {[0.5,0.5]} & {[0.4,0.5]} \\
x & x & x & {[0.5,0.5}
\end{array}\right] ; \\
{ }_{3} \tilde{B}^{(3)} & =\left[\begin{array}{cccc}
{[0.5,0.5]} & x & x & x \\
{[0.4,0.5]} & {[0.5,0.5]} & x & x \\
{[0.3,0.5]} & x & {[0.5,0.5]} & x \\
{[0.4,0.7]} & x & x & {[0.5,0.5]}
\end{array}\right] .
\end{aligned}
$$

Incomplete interval additive preference relation for the four candidates with respect to the conformance quality criterion:

$$
\begin{aligned}
{ }_{4} \tilde{B}^{(1)} & =\left[\begin{array}{cccc}
{[0.5,0.5]} & {[0.2,0.4]} & x & x \\
x & {[0.5,0.5]} & {[0.7,0.8]} & x \\
x & x & {[0.5,0.5]} & {[0.3,0.4]} \\
x & x & x & {[0.5,0.5]}
\end{array}\right] ; \\
{ }_{4} \tilde{B}^{(2)} & =\left[\begin{array}{cccc}
{[0.5,0.5]} & x & x & x \\
x & {[0.5,0.5]} & x & x \\
x & x & {[0.5,0.5]} & x \\
{[0.2,0.4]} & {[0.4,0.6]} & {[0.5,0.6]} & {[0.5,0.5]}
\end{array}\right] ; \\
{ }_{4} \tilde{B}^{(3)} & =\left[\begin{array}{cccc}
{[0.5,0.5]} & x & x & x \\
{[0.6,0.7]} & {[0.5,0.5]} & {[0.5,0.6]} & {[0.6,0.8]} \\
x & x & {[0.5,0.5]} & x \\
x & x & x & {[0.5,0.5]}
\end{array}\right] .
\end{aligned}
$$

Incomplete interval additive preference relation for the four candidates with respect to the conflict resolution criterion:

$$
{ }_{5} \tilde{B}^{(1)}=\left[\begin{array}{cccc}
{[0.5,0.5]} & x & x & x \\
{[0.5,0.7]} & {[0.5,0.5]} & {[0.8,0.9]} & {[0.4,0.7]} \\
x & x & {[0.5,0.5]} & x \\
x & x & x & {[0.5,0.5]}
\end{array}\right] ;
$$




$$
\begin{aligned}
{ }_{5} \tilde{B}^{(2)} & =\left[\begin{array}{cccc}
{[0.5,0.5]} & {[0.2,0.4]} & x & x \\
x & {[0.5,0.5]} & {[0.7,0.8]} & x \\
x & x & {[0.5,0.5]} & {[0.2,0.3]} \\
x & x & x & {[0.5,0.5]}
\end{array}\right] ; \\
{ }_{5} \tilde{B}^{(3)} & =\left[\begin{array}{cccc}
{[0.5,0.5]} & x & {[0.4,0.7]} & x \\
x & {[0.5,0.5]} & {[0.6,0.7]} & x \\
x & x & {[0.5,0.5]} & x \\
x & x & {[0.8,0.9]} & {[0.5,0.5]}
\end{array}\right] .
\end{aligned}
$$

Utilize the procedure proposed in Section 4, the collective interval additive preference relations for five criteria and its priority weights are shown in Table 1. All the collective interval additive preference relations and their priority ratings with respect to each criterion are shown in Table 2. The overall priority ratings for each alternative appear in the last column of Table 3, which are $\tilde{Z}_{1}=[0.139,0.56], \tilde{Z}_{2}=[0.119,0.449], \tilde{Z}_{3}=[0.126,0.471], \tilde{Z}_{4}=[0.121,0.509]$. In order to rank the alternatives, by Eq. (28), we construct the possibility degree matrix:

$$
P=\left[\begin{array}{cccc}
0.5 & 0.588 & 0.566 & 0.543 \\
0.412 & 0.5 & 0.477 & 0.456 \\
0.434 & 0.522 & 0.5 & 0.478 \\
0.457 & 0.544 & 0.522 & 0.5
\end{array}\right] \text {. }
$$

Then by the normalizing rank aggregation method Eq. (29), we have:

$$
\varpi=(0.275,0.231,0.242,0.253)^{T}
$$

and thus $\varpi_{1}>\varpi_{4}>\varpi_{3}>\varpi_{2}$, the ranking order of four suppliers is $x_{1} \succ x_{4} \succ x_{3} \succ x_{2}$, and hence $x_{1}$ is the best supplier.

Remark 1. In the two above examples, we can see that we only need to pride $n-1$ pairwise values, then we can construct the consistent additive interval fuzzy preference relations. Furthermore, the $n-1$ values are not just $\left\{r_{12}, r_{23}, \ldots, r_{n-1 n}\right\}$, while the existing methods must be $\left\{r_{12}, r_{23}, \ldots, r_{n-1 n}\right\}$ (Wang, Chen 2008). The existing methods only deal with the crisp numbers, while in this paper, we consider more uncertain environments, i.e. incomplete interval fuzzy preference relations.

Table 1. Collective interval additive preference relations for five criteria and its priority weights

\begin{tabular}{ccccccc}
\hline & $c_{1}$ & $c_{2}$ & $c_{3}$ & $c_{4}$ & $c_{5}$ & Priority weights \\
\hline$c_{1}$ & {$[0.5,0.5]$} & {$[0.27,0.4]$} & {$[0.28,0.53]$} & {$[0.12,0.56]$} & {$[0.1,0.57]$} & {$[0.085,0.257]$} \\
$c_{2}$ & {$[0.6,0.73]$} & {$[0.5,0.5]$} & {$[0.51,0.63]$} & {$[0.35,0.66]$} & {$[0.33,0.67]$} & {$[0.152,0.32]$} \\
$c_{3}$ & {$[0.47,0.72]$} & {$[0.37,0.49]$} & {$[0.5,0.5]$} & {$[0.34,0.53]$} & {$[0.32,0.54]$} & {$[0.133,0.278]$} \\
$c_{4}$ & {$[0.44,0.88]$} & {$[0.34,0.65]$} & {$[0.47,0.66]$} & {$[0.5,0.5]$} & {$[0.48,0.51]$} & {$[0.149,0.32]$} \\
$c_{5}$ & {$[0.43,0.9]$} & {$[0.33,0.67]$} & {$[0.46,0.68]$} & {$[0.49,0.52]$} & {$[0.5,0.5]$} & {$[0.147,0.328]$} \\
\hline
\end{tabular}


Table 2. Collective interval additive preference relations and their priority ratings

\begin{tabular}{|c|c|c|c|c|c|}
\hline & $x_{1}$ & $x_{2}$ & $x_{3}$ & $x_{4}$ & priority ratings \\
\hline \multicolumn{6}{|l|}{$c_{1}$} \\
\hline$x_{1}$ & {$[0.5,0.5]$} & {$[0.73,0.83]$} & {$[0.47,0.7]$} & {$[0.5,0.87]$} & {$[0.238,0.429]$} \\
\hline$x_{2}$ & {$[0.17,0.27]$} & {$[0.5,0.5]$} & {$[0.23,0.37]$} & {$[0.27,0.53]$} & {$[0.126,0.246]$} \\
\hline$x_{3}$ & {$[0.3,0.53]$} & {$[0.63,0.77]$} & {$[0.5,0.5]$} & {$[0.53,0.67]$} & {$[0.213,0.365]$} \\
\hline$x_{4}$ & {$[0.13,0.5]$} & {$[0.47,0.73]$} & {$[0.3,0.47]$} & {$[0.5,0.5]$} & {$[0.155,0.325]$} \\
\hline \multicolumn{6}{|l|}{$c_{2}$} \\
\hline$x_{1}$ & {$[0.5,0.5]$} & {$[0.8,0.87]$} & {$[0.53,0.7]$} & {$[0.6,0.9]$} & {$[0.270,0.424]$} \\
\hline$x_{2}$ & {$[0.13,0.2]$} & {$[0.5,0.5]$} & {$[0.23,0.33]$} & {$[0.3,0.53]$} & {$[0.130,0.224]$} \\
\hline$x_{3}$ & {$[0.3,0.47]$} & {$[0.67,0.77]$} & {$[0.5,0.5]$} & {$[0.57,0.7]$} & {$[0.226,0.348]$} \\
\hline$x_{4}$ & {$[0.1,0.4]$} & {$[0.47,0.7]$} & {$[0.3,0.43]$} & {$[0.5,0.5]$} & {$[0.152,0.291]$} \\
\hline \multicolumn{6}{|l|}{$c_{3}$} \\
\hline$x_{1}$ & {$[0.5,0.5]$} & {$[0.57,0.67]$} & {$[0.37,0.57]$} & {$[0.4,0.67]$} & {$[0.206,0.338]$} \\
\hline$x_{2}$ & {$[0.33,0.43]$} & {$[0.5,0.5]$} & {$[0.3,0.4]$} & {$[0.33,0.5]$} & {$[0.165,0.258]$} \\
\hline$x_{3}$ & {$[0.43,0.63]$} & {$[0.6,0.7]$} & {$[0.5,0.5]$} & {$[0.53,0.6]$} & {$[0.232,0.343]$} \\
\hline$x_{4}$ & {$[0.33,0.6]$} & {$[0.5,0.67]$} & {$[0.4,0.47]$} & {$[0.5,0.5]$} & {$[0.195,0.312]$} \\
\hline \multicolumn{6}{|l|}{$c_{4}$} \\
\hline$x_{1}$ & {$[0.5,0.5]$} & {$[0.4,0.5]$} & {$[0.47,0.67]$} & {$[0.4,0.7]$} & {$[0.196,0.338]$} \\
\hline$x_{2}$ & {$[0.5,0.6]$} & {$[0.5,0.5]$} & {$[0.57,0.67]$} & {$[0.5,0.7]$} & {$[0.230,0.352]$} \\
\hline$x_{3}$ & {$[0.33,0.53]$} & {$[0.33,0.43]$} & {$[0.5,0.5]$} & {$[0.43,0.53]$} & {$[0.178,0.286]$} \\
\hline$x_{4}$ & {$[0.3,0.6]$} & {$[0.3,0.5]$} & {$[0.47,0.57]$} & {$[0.5,0.5]$} & {$[0.174,0.310]$} \\
\hline \multicolumn{6}{|l|}{$c_{5}$} \\
\hline$x_{1}$ & {$[0.5,0.5]$} & {$[0.27,0.47]$} & {$[0.47,0.77]$} & {$[0.1,0.53]$} & {$[0.142,0.343]$} \\
\hline$x_{2}$ & {$[0.53,0.73]$} & {$[0.5,0.5]$} & {$[0.7,0.8]$} & {$[0.33,0.57]$} & {$[0.220,0.394]$} \\
\hline$x_{3}$ & {$[0.23,0.53]$} & {$[0.2,0.3]$} & {$[0.5,0.5]$} & {$[0.13,0.27]$} & {$[0.114,0.242]$} \\
\hline$x_{4}$ & {$[0.47,0.9]$} & {$[0.43,0.67]$} & {$[0.73,0.87]$} & {$[0.5,0.5]$} & {$[0.227,0.444]$} \\
\hline
\end{tabular}

Table 3. Final overall ratings

\begin{tabular}{|c|c|c|c|c|c|c|}
\hline & $c_{1}$ & $c_{2}$ & $c_{3}$ & $c_{4}$ & $c_{5}$ & Final ratings \\
\hline Criteria weight & {$[0.085,0.257]$} & {$[0.152,0.32]$} & {$[0.133,0.278]$} & {$[0.149,0.32]$} & {$[0.147,0.328]$} & \\
\hline$x_{1}$ & {$[0.238,0.429]$} & {$[0.270,0.424]$} & {$[0.206,0.338]$} & {$[0.196,0.338]$} & {$[0.142,0.343]$} & {$[0.139,0.560]$} \\
\hline$x_{2}$ & {$[0.126,0.246]$} & {$[0.130,0.224]$} & {$[0.165,0.258]$} & {$[0.230,0.352]$} & {$[0.220,0.394]$} & {$[0.119,0.449]$} \\
\hline$x_{3}$ & {$[0.213,0.365]$} & {$[0.226,0.348]$} & {$[0.232,0.343]$} & {$[0.178,0.286]$} & {$[0.114,0.242]$} & {$[0.126,0.471]$} \\
\hline$x_{4}$ & {$[0.155,0.325]$} & {$[0.152,0.291]$} & {$[0.195,0.312]$} & {$[0.174,0.310]$} & {$[0.227,0.444]$} & {$[0.121,0.509]$} \\
\hline
\end{tabular}




\section{Conclusions}

In this paper, we have extended a characterization of the consistency property defined by the additive transitivity property of the fuzzy preference. Then, we extend this characterization to the interval fuzzy preference relation which is based on the additive transitivity property. Using the characterization, we proposed a method to construct interval additive consistent fuzzy preference relations from a set of $n-1$ preference data. The study reveals that the proposed method can reduce the number of pairwise comparisons. Our first illustrative example involves seven alternatives requiring six comparison judgments. Therefore, the number of comparison can be reduced by $C_{7}^{2}-6=15$ times. Our second example which involves three decision makers, four alternatives and five criteria, can be reduced the number of comparisons by $3 \times\left(C_{5}^{2}-4+5 \times\left(C_{4}^{2}-3\right)\right)=63$ times, while all the preference relations maintain the additive and consistency. We also present a procedure to deal with the analytic hierarchy problem for group decision making with incomplete interval fuzzy preference relations. The procedure contains three parts: (1) Determine the priority weights of criteria; (2) Determine the priority ratings for each alternative with respect to each criterion; (3) Obtaining the priority weight for each alternative. Finally, we apply the procedure to a practical application for supplier selection in supply chain management.

In the future, we would study the incomplete linguistic interval fuzzy preference relations (Xu, Da 2010), and other applications (Keršulienė, Turskis 2011; Napalkova, Merkuryeva 2012; Wu et al. 2012).

\section{Acknowledgements}

The authors is very grateful to three anonymous referees for their insightful and constructive comments and suggestions that have led to an improved version of this paper. This work was supported by the National Natural Science Foundation of China (NSFC) under Grant 71101043, and the major project of the national Social Science Fund of China under Grant 12\&ZD214, the International Academic Cooperation Team Development Foundation of Business School, Hohai University under Grant UAH-2011-181.

\section{References}

Alonso, S.; Chiclana, F.; Herrera, F.; Herrera-Viedma, E.; Alcalá-Fdez, J.; Porcel, C. 2008. A consistencybased procedure to estimate missing pairwise preference values, International Journal of Intelligent Systems 23(2): 155-175. http://dx.doi.org/10.1002/int.20262

Arbel, A. 1989. Approximate articulation of preference priority derivation, European Journal of Operational Research 43(3): 317-326. http://dx.doi.org/10.1016/0377-2217(89)90231-2

Chen, C. T.; Lin, C. T.; Huang, S. F. 2006. A fuzzy approach for supplier evaluation and selection in supply chain management, International Journal of Production Economics 102(2): 289-301. http://dx.doi.org/10.1016/j.ijpe.2005.03.009

Chiclana, F.; Herrera-Viedma, E.; Alonso, S.; Herrera, F. 2008. A note on the estimation of missing pairwise preference values: a U-consistency based method, International Journal of Uncertainty, Fuzziness and Knowledge-Based Systems 16(2): 19-32. http://dx.doi.org/10.1142/S0218488508005467 
Csutora, R.; Buckley, J. J. 2001. Fuzzy hierarchical analysis: the Lambda-Max method, Fuzzy Sets and Systems 120(2): 181-195. http://dx.doi.org/10.1016/S0165-0114(99)00155-4

Fedrizzi, M.; Silvio, G. 2007. Incomplete pairwise comparison and consistency optimization, European Journal of Operational Research 183(1): 303-313. http://dx.doi.org/10.1016/j.ejor.2006.09.065

Herrera-Viedma, E.; Herrera, F.; Chiclana, F.; Luque, M. 2004. Some issues on consistency of fuzzy preference relations, European Journal of Operational Research 154(1): 98-109. http://dx.doi.org/10.1016/S0377-2217(02)00725-7

Herrera-Viedma, E.; Alonso, S.; Chiclana, F.; Herrera, F. 2007a. A consensus model for group decision making with incomplete fuzzy preference relations, IEEE Transactions on Fuzzy Systems 15(5): 863-877. http://dx.doi.org/10.1109/TFUZZ.2006.889952

Herrera-Viedma, E.; Chiclana, F.; Herrera, F.; Alonso, S. 2007b. Group decision-making model with incomplete fuzzy preference relations based on additive consistency, IEEE Transactions on Systems, Man, and Cybernetics-Part B: Cybernetics 37(1): 176-189. http://dx.doi.org/10.1109/TSMCB.2006.875872

Herrera, F.; Martíze, L.; Sánchez, P. J. 2005. Managing non-homogeneous information in group decision making, European Journal of Operational Research 166: 115-132.

http://dx.doi.org/10.1016/j.ejor.2003.11.031

Islam, R.; Biswal, M. P.; Alam, S. S. 1997. Preference programming and inconsistent interval judgments, European Journal of Operational Research 97(1): 53-62.

http://dx.doi.org/10.1016/S0377-2217(95)00377-0

Jiang, Y. L. 2007. An approach to group decision making based on interval fuzzy relations, Journal of Systems Science and Systems Engineering 16(1): 113-120. http://dx.doi.org/10.1007/s11518-006-5026-2

Kacprzyk, J. 1986. Group decision making with a fuzzy majority, Fuzzy Sets and Systems 18(2): 105-118. http://dx.doi.org/10.1016/0165-0114(86)90014-X

Keršuliene, V.; Turskis, Z. 2011. Integrated fuzzy multiple criteria decision making model for architect selection, Technological and Economic Development of Economy 17(4): 645-666.

http://dx.doi.org/10.3846/20294913.2011.635718

Kress, M. 1991. Approximate articulation of preference and priority derivation-a comment, European Journal of Operational Research 52(3): 382-383. http://dx.doi.org/10.1016/0377-2217(91)90174-T

Liu, X. W.; Pan, Y. W.; Xu, Y. J.; Yu, S. 2012. Least square completion and inconsistency repair methods for additively consistent fuzzy preference relations, Fuzzy Sets and Systems 198: 1-19. http://dx.doi.org/10.1016/j.fss.2011.11.009

Mikhailov, L. 2002. Fuzzy analytical approach to partnership selection in formation of virtual enterprises, Omega 30(5): 393-401. http://dx.doi.org/10.1016/S0305-0483(02)00052-X

Napalkova, L.; Merkuryeva, G. 2012. Multi-objective stochastic simulation-based optimisation applied to supply chain planning, Technological and Economic Development of Economy 18(1): 132-148. http://dx.doi.org/10.3846/20294913.2012.661190

Saaty, T. L. 1980. The analytic hierarchy process. New York: McGraw-Hill. 287 p.

Saaty, T. L.; Vargas, L. G. 1987. Uncertainty and rank order in the analytic hierarchy process, European Journal of Operational Research 32(1): 107-117. http://dx.doi.org/10.1016/0377-2217(87)90275-X

Tanino, T. 1984. Fuzzy preference orderings in group decision making, Fuzzy Sets and Systems 12(2): 117-131. http://dx.doi.org/10.1016/0165-0114(84)90032-0

Vaidya, O. S.; Kumar, S. 2006. Analytical hierarchy process: an overview of applications, European Journal of Operational Research 169(1): 1-29. http://dx.doi.org/10.1016/j.ejor.2004.04.028

Wang, T. C.; Chen, Y. H. 2007. Applying consistent fuzzy preference relations to partnership selection, Omega 35(4): 384-388. http://dx.doi.org/10.1016/j.omega.2005.07.007 
Wang, T. C.; Chen, Y. H. 2008. Applying fuzzy linguistic preference relations to the improvement of consistency of fuzzy AHP, Information Sciences 178(19): 3755-3765. http://dx.doi.org/10.1016/j.ins.2008.05.028

Wang, Y. M.; Yang, J. B.; Xu, D. L. 2005. A two-stage logarithmic goal programming method for generating weights from interval comparison matrices, Fuzzy Sets and Systems 152(3): 475-498.

http://dx.doi.org/10.1016/j.fss.2004.10.020

Wang, Y. M. 2006. On lexicographic goal programming method for obtaining interval weights from an interval comparison matrix, Applied Mathematics and Computation 173(2): 985-991. http://dx.doi.org/10.1016/j.amc.2005.04.029

Wang, Y. M.; Chin, K. S. 2006. An eigenvector method for generating normalized interval and fuzzy weights, Applied Mathematics and Computation 181(2): 1257-1275.

http://dx.doi.org/10.1016/j.amc.2006.02.026

Wang, Y. M.; Elhag, T. M. S. 2007. A goal programming method for obtaining interval weights from an interval comparison matrix, European Journal of Operational Research 177(1): 458-471.

http://dx.doi.org/10.1016/j.ejor.2005.10.066

Wu, W. S.; Kou, G.; Peng, Y.; Ergu, D. 2012. Improved AHP-group decision making for investment strategy selection, Technological and Economic Development of Economy 18(2): 299-316. http://dx.doi.org/10.3846/20294913.2012.680520

Xu, Y. J.; Da, Q. L.; Liu, L. H. 2009. Normalizing rank aggregation method for priority of a fuzzy preference relation and its effectiveness, International Journal of Approximate Reasoning 50(8): 1287-1297. http://dx.doi.org/10.1016/j.ijar.2009.06.008

$\mathrm{Xu}$, Y. J. 2010. Note on "The induced continuous ordered weighted geometric operators and their application in group decision making", Computers \& Industrial Engineering 59(2): 365-366.

http://dx.doi.org/10.1016/j.cie.2010.05.006

Xu, Y. J.; Da, Q. L. 2010. Standard and mean deviation methods for linguistic group decision making and their applications, Expert Systems with Applications 37(8): 5905-5912. http://dx.doi.org/10.1016/j.eswa.2010.02.015

Xu, Y. J.; Da, Q. L.; Wang, H. M. 2010. A note on group decision-making procedure based on incomplete reciprocal relations, Soft Computing 15(7): 1289-1300. http://dx.doi.org/10.1007/s00500-010-0662-3

Xu, Y. J.; Patnayakuni, R.; Wang, H. M. 2013. Logarithmic least squares method to priority for group decision making with incomplete fuzzy preference relations, Applied Mathematical Modelling 37(4): 2139-2152. http://dx.doi.org/10.1016/j.apm.2012.05.010

Xu, Z. S. 2004a. On compatibility of interval fuzzy preference relations, Fuzzy Optimization and Decision Making 3(3): 217-225. http://dx.doi.org/10.1023/B:FODM.0000036864.33950.1b

$\mathrm{Xu}$, Z. S. 2004b. Goal programming models for obtaining the priority vector of incomplete fuzzy preference relation, International Journal of Approximate Reasoning 36(3): 261-270. http://dx.doi.org/10.1016/j.ijar.2003.10.011

$\mathrm{Xu}, \mathrm{Z}$. S. 2005. A procedure for decision making based on incomplete fuzzy preference relation, Fuzzy Optimization and Decision Making 4(3): 175-189. http://dx.doi.org/10.1007/s10700-005-1887-y

$\mathrm{Xu}, \mathrm{Z}$. S.; Chen, J. 2008. Some methods for deriving the priority weights from interval fuzzy preference relations, European Journal of Operational Research 184(1): 266-280.

http://dx.doi.org/10.1016/j.ejor.2006.11.011

Yejun XU. Doctor, Associate Professor at the State Key Laboratory of Hydrology-Water Resources and Hydraulic Engineering, and Business School, Hohai University. He has contributed over 50 journal articles to professional journals such as Fuzzy Sets and Systems, Information Sciences, International Journal of Approximate Reasoning, Knowledge-Based Systems, etc. His current research interests include multi-criteria decision making, computing with words, and information sciences. 
Ravi PATNAYAKUNI. Doctor, Associate Professor at the Department of Economics and IS, University of Alabama in Huntsville. He has contributed over 20 journal papers to professional journals such as Information Systems Frontiers, Decision Support Systems, IEEE Transactions on Engineering Management, etc. His current research interests include supply chain management, knowledge management and systems development.

Feifei TAO. Doctor, Lecturer at Business School, Hohai University. His current research interests include information system.

Huimin WANG. Doctor, Professor at the State Key Laboratory of Hydrology-Water Resources and Hydraulic Engineering, and Business School, Hohai University. She has contributed over 100 journal papers to professional journals. Her current research interests include supply chain management, water resource management. 